\title{
An Effective Neighborhood Initial-Playback Based Caching Scheme for Video on Demand over Mobile Ad Hoc Network
}

\author{
Saleh Ali Alomari, Member, IACSIT, Vaithegy Doraisamy, and Putra Sumari
}

\begin{abstract}
Video on demand (VOD) System over mobile Ad hoc network (MANET) is a system that provides video services to mobile clients. The main problem in these systems is the high service delay where the mobile clients have to wait to watch their favorite movie. The importance of this study is to find a solution on how to reduce the delay time in the system. This paper proposed a novel caching scheme called neighborhood-initial playback based caching scheme (NIPBCS) to overcome the service delay by sending the 1st segment of a movie to the new client by a local forwarder (LF). The LFs will send the 1st segment of the movie selected to the client's First buffer when the new client's arrival is detected. When the client wants to watch another movie, the client can tune in to the broadcasting channel that will broadcast the 1st segment of the movie or the client can request from the neighbors or otherwise if the movie the client wants to watch is the same as the 1st segment sent by the Local Forwarder, the client can just switch to the First Buffer and start watching the movie. The aim of this caching scheme is to reduce the time delay in VOD system over MANET. The result obtained proves that NIPBCS reduces the time delay of the system when the number of clients increases and the client failure probability is reduced when the number of clients increases.
\end{abstract}

Index Terms-Video on demand, MANET, NIPBCS, staggered broadcast

\section{INTRODUCTION}

In recent years, Video on Demand service has become more popular and many VOD service applications are emerging. In recent years, Video-On-Demand service has become more and more popular and many VOD service applications are emerging. Peoples nowadays do not prefer to waste their time but wants to occupy the time by doing something useful like watching their favorite movie when they have spare time. For instance, while waiting turn to finish their task in post office, airport, immigration office, school campus and restaurants, etc. Most of them wished to watch their favorite video using their mobile devices such as hand phones, laptops and PDAs. With the development in wireless technology such as IEEE 802.11, their wish now can become true.

The production of $3 \mathrm{G}$ and $4 \mathrm{G}$ smart phones such as iPhone,

Manuscript received April 12, 2021; revised June 30, 2012. This project is part of a larger research project in the MCRG and this work was supported by Universiti RU grant (1001 / PKOMP/ 817066) from the Universiti Sains Malaysia.

The authors are with the Multimedia Computing Research Group, School of Computer Science, Universiti Sains Malaysia, 11800 Pulau Penang, Malaysia (e-mail: salehalomari2005@yahoo.com, vaiwoods@yahoo.com, putras@cs.usm.my).
HTC EVO 4G and PDAs are increasing every year and the price of the devices are also affordable. The main reason to this increment in production is because of the new high technology applications introduced in it especially the application that enables the users to download and watch movie in their phone. Now all the smart phone mobile users can join the Video on Demand System over MANET network and stream or download their favorite movie at anytime and at anywhere.

In Video on Demand System over MANET network, the mobile phone clients can download videos to their mobiles from a video server that is builds in a Mobile Ad Hoc Network (MANET) environment [1]. In the MANET environment, it has a collection of two or more devices with wireless communications and can communicate with each other without the aid of centralized administrator [2] and the mobile clients does not have a fixed topology, thus it can enter and leave the network easily [3].

This paper proposes a novel caching scheme called Neighborhood-Initial Playback Based Caching Scheme (NIPBCS) for video on demand system over MANET to reduce the time delay. This paper organized as follows: section II related backgrounds and section III described the proposed work.

\section{RELATED BACKGROUND}

This section discusses about the Video on Demand system over MANET, Staggered broadcast protocol and types of caching schemes used in VOD systems.

\section{A. Video on Demand System over MANET}

There are many VOD systems over MANET are proposed to reduce the waiting time for the mobile clients to get the video requested. MobiVoD is an example of VOD System over MANET where the system is classified to three components, the server as a main provider for the videos, Local Forwarder as a disseminate point of video to mobile clients, and mobile clients as a subscriber who receives and watches video. MANET environment enable the mobile clients to communicate and connect with each other through direct wireless links such as IEEE 802.11. All the videos will be stored in the main server and Local Forwarder (LF) is used to receive videos from the server and then broadcast the videos as segments through the wireless interface card to mobile clients. The server and the Local Forwarder are interconnected via an infrastructure-based wireless network. The local forwarder provides the services to the mobile clients within the coverage area, in the same time the mobile clients 
can request and receive videos directly from the Local Forwarder. Staggered Broadcasting (SB) Protocol is used in this system to disseminate the video data to the mobile clients.

\section{B. Staggered Broadcast Protocol}

Staggered Broadcasting protocol [4], [5] is used in VOD system over MANET to segment the video and this protocol requires the client to join only 1 channel as shown in Fig. 1. The server bandwidth will be divided into equally sized channels and the videos also will be segmented into equally sized segments. The divided channels and the segmented video's value are equal. Mobile clients who are in the local forwarder's coverage area can join the channels to receive the broadcasted segments of videos and can start watching the movie.

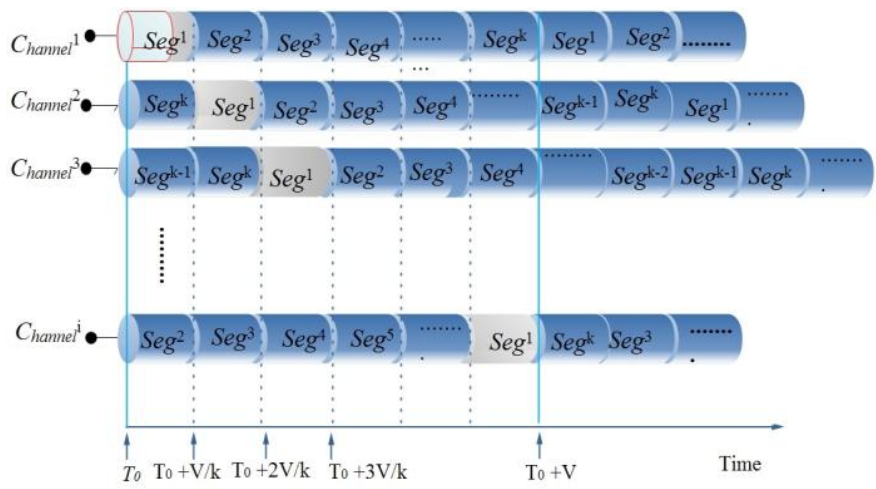

Fig. 1. Staggered broadcast protocol.

\section{Caching Schemes}

Caching schemes are introduced in the VOD over MANET to reduce the time delay, bandwidth reduction, request rejection and save caching space in the system. There are many caching schemes proposed by the researchers such as:

- Partial Video Sequence (PVS) caching scheme [6]: It decomposes video sequences into a number of parts by using a scalable video compression algorithm. Video parts are selected to be cached in local video servers based on the amount of bandwidth that would be demanded from the distribution network and central video server if it was only kept in the central video server. PVC caching improves the performance of caching, provides more reduction in the bandwidth requirement than the conventional caching scheme.

- Prefix Caching [7], it could reduce the request rejection ratio and client waiting time. The network bandwidth usage also has been reduced by the caching scheme through sharing the video data of the currently played video object with other clients of the active chain.

- Dominating-Set Cache (DSC) [8] In DSC, when a new client $\mathrm{X}$ is in the transmission range of existing client $\mathrm{Y}$ and the client is not forwarding cache to any other client, client $\mathrm{X}$ can make use of the cache at $\mathrm{Y}$ and plays the video. But if client $X$ is not in the transmission area of client $\mathrm{Y}$, the client $\mathrm{Y}$ will find another client $\mathrm{Z}$ who shares the same transmission area with itself and client $\mathrm{X}, \mathrm{Y}$ will download the broadcast portion that $\mathrm{x}$ misses from $\mathrm{Z}$ and forwards it to $\mathrm{X}$.
- Random Cache [9], simplest selective cache algorithm and only a number of selected clients are allowed to cache the first segment of a video. The advantage of this caching scheme is the saving on caching space and the disadvantage is it has the higher service delay.

- Client Caching Scheme for Interactive Videoon-Demand [10], it is used to reduce the traffic load on the network where a local client will cache a video and when the video once retrieved from the server, it will be retained in a video cache on the client for the remainder of the session. If other clients want to watch the same video again, the video can be viewed from the cache and not from the server. If the local cache is full, an aging mechanism is used to decide which frame is to be overwritten by the most recently requested one.

- Neighbor Based Caching Scheme [11], Mobile client can download the first segment of the video from the initial buffer of the neighbor client to its own initial buffer. The mobile client must be within the coverage area of neighbor client. When the initial buffer of the mobile client is filled with the first segment downloaded from the neighbor client, now it will acts as the self based caching scheme and broadcasts the segment like how the Self based caching system does.

\section{PROPOSED WORK}

\section{A. Overview of Neighborhood Initial-Playback Based Caching Scheme (IPBCS)}

The new caching scheme proposed in this paper is called Neighborhood Initial-Playback Based Caching Scheme (IPBCS). This caching scheme is used to reduce waiting time or the service latency of the mobile client to make the viewer's waiting time is less than that of previous scheme mechanism.

In IPBCS, a new client will receive the 1st segment of a movie from the local forwarder upon the detection of the arrival of the client to make sure all the clients in the MANET network area will have the 1 st segment of (movie $j$ ) from the closer local forwarder.

\section{1) Features of Mobile Client}

Mobile clients involved in this research are $3 \mathrm{G}$ and $4 \mathrm{G}$ smart user phones because this type of mobile clients can download and playback videos to their phones. The memory card of these mobile clients is divided into three types of buffer, First buffer, Initial Buffer, and Pre-fetched buffer as shown in Fig. 2. The First Buffer is to store the 1st segment of a movie that was sent by the Local Forwarder. The Initial buffer is to cache the 1st segment of a movie from the Staggered Broadcasting Protocol channels or from the neighbors meanwhile Pre-fetched buffer is to cache the second segment and the following segments of the movie from the Staggered Broadcasting Protocol channels. The size of First buffer and Initial buffer is equal to the size of the 1st segment of a movie meanwhile the size of the pre-fetched buffer is larger than both buffers. 


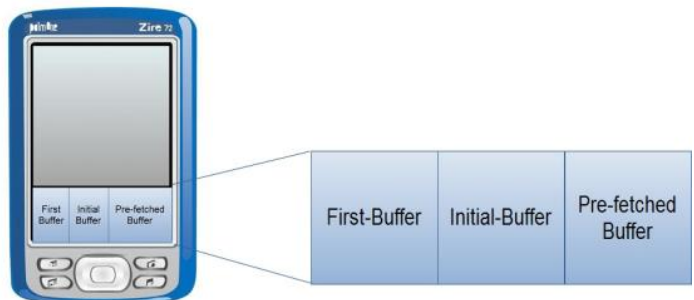

Fig. 2. The feature of mobile client with 3 buffers.

\section{2) Features of IPBCS}

There are few stages a new client A has to go through to receive the 1st segment of a movie. First the local forwarder will detect the arrival of the new client and will send a message to the client to accept the 1st segment as shown in Fig 3 and then will send the 1 st segment of movie A to the client's first buffer as shown in Fig. 4.

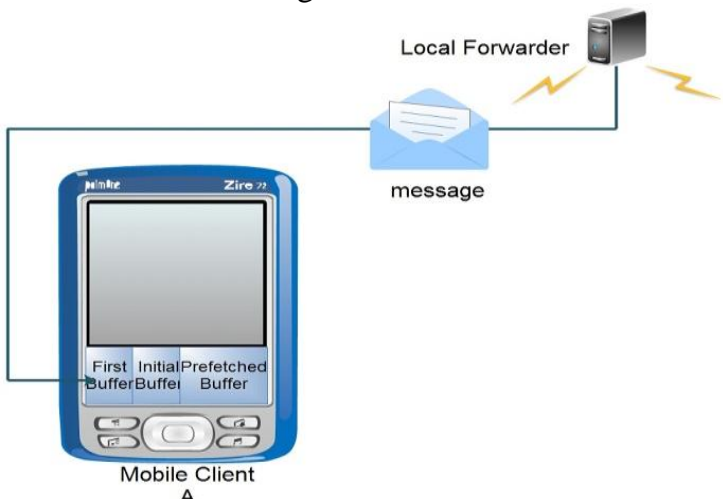

Fig. 3. Mobile client a receiving message from local forwarder.

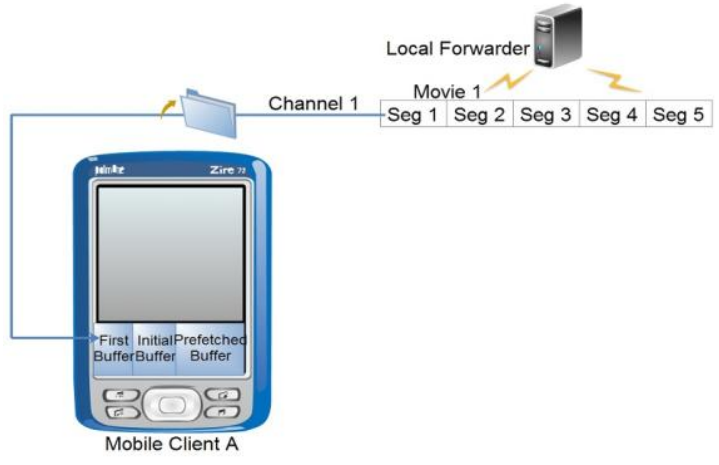

Fig. 4. Mobile Client A receiving First Segment of Movie 1.

Client_Detection:

LFposition-Client_iposition $\leq 100 \mathrm{~m}$

a) Scenario of Detection Procedure for Mobile Client Arrival:

1. U1 enters the network

2. LF1 detects the arrival of $\mathrm{U} 1$

3. LF1 checks which movie's 1st segment is not in other client's first buffer.

4. If all the movie's 1 st segment is in all the other client's $1 \mathrm{st}$ buffer

- LF1 will send again 1st segment of any movie to U1

5. Else if any movie's 1st segment is still not sent

- LF1 will chose that 1 st segment to be sent to U1

6. LF1 sends a message to the U1 to accept the 1st segment.

7. LF1 sends the 1st segment to U1's 1st buffer.

8. U1 accepts the 1st segment.

Where, U1 is the mobile client and LF1 is Local Forward 1.
Fig. 5 shows the architecture of clients getting $1^{\text {st }}$ segment of movies from the Local Forwarder when the clients are at the Local Forwarder's service area.

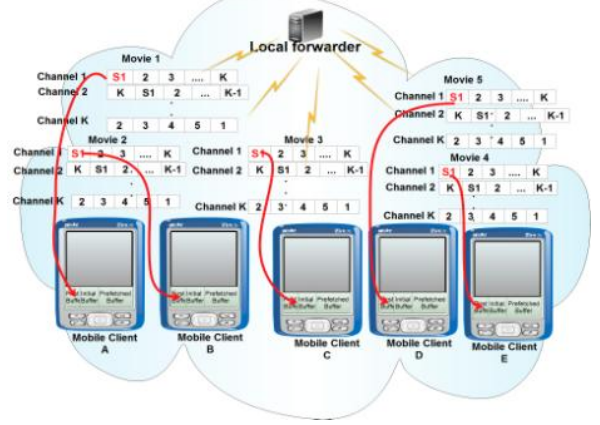

Fig. 5. The architecture of clients receiving 1st segment of movies from local forwarder.

The client then will send a message to local forwarder to acknowledge that it already received the 1 st segment. At the same time, the new client can request for 1st segment of its favorite movie to the local forwarder and can tune in to the broadcasting channel that is broadcasting the 1st segment the soonest. But if the 1st segment that was sent to the first buffer of the new client is the same as the movie that the new client wished to watch, the client can immediately start watching the 1st segment from the First Buffer. Shown below is the scenario of playback procedure when the mobile clients (U1) already have the first segment of movie A in its First Buffer.

b) Scenario of Playback Procedure When the Clinet Already Have the 1st Segment in the First Buffer:

1. LF detect mobile client $\mathrm{U} 1$

2. LF send message followed by S1 to U1 First Buffer

3. $\mathrm{U} 1$ request 1 st segment of movie 1

- If First Buffer have the same 1st segment of movie 1

4. U1 playback the 1st segment from the First Buffer.

5. At the same time U1 join the broadcasting channel to receive the rest of segments in the pre-fetched buffer.

6. After U1 finish viewing the 1st segment will switch to the pre-fetched buffer.

7. Quit the channel.

Where, U1 is the mobile client and LF1 is Local Forward 1.

If the 1 st segment of the movie is not the same, and the client request time is trequest, (trequest $=t 0, t 0+s, t 0+2 s, t 0+3 s, t 0+4 s$ or $t 0+V)$, the new mobile client can tune in either to channel 1, channel 2, channel 3, channel 4 or channel 5 of the Staggered Broadcasting Protocol.

c) Scenario of Playback Procedure When the Clinet Request from $L F$ :

1. Detect local forwarder

2. Find the channel from local forwarder which is going to broadcast the $\mathrm{S} 1$ soonest

3. Wait and join that channel

4. Download the S1 in its Initial_play buffer and cache remaining segments into pre-fetched buffer.

5. Play $S 1$ in Initial_play buffer and switch to pre-fetched buffer to play remaining segments.

6. Quit the channel.

Where, S1 is First segment. 
But, if the request time: (trequest $=t 0,+\delta$, $t 0+s+\delta, t 0+2 s+\delta, t 0+3 s+\delta, t 0+4 s+\delta \quad 0 r \quad t 0+V+\delta), \quad$ where $(0<\delta<s)$, client can request for the 1 st segment from the neighbors.

At this stage, the Local Forwarder will send a message to the new client informing that the client has missed the broadcasting time of the 1st segment. It also sends information about neighbors who have the 1 st segment and the position of the neighbors with the time delay for the broadcasting protocol to broadcast the same segment again. The new client can now send message to its neighbors who have the 1st segment when the distance of the neighbor is:

$$
\text { Client }[i] \text { position-Client }[i+1] \text { position } \leq 20 \mathrm{~m}
$$

Then neighbors who have the 1st segment in its first buffer will reply the request and the new client can accept the 1st segment from the neighbor who is either nearer to it or not busy forwarding any segment to other clients meanwhile can cache the rest of the segments into its pre-fetched buffer by tuning to the broadcasting channel that broadcast the 2 nd segment the soonest as shown in Fig. 6.

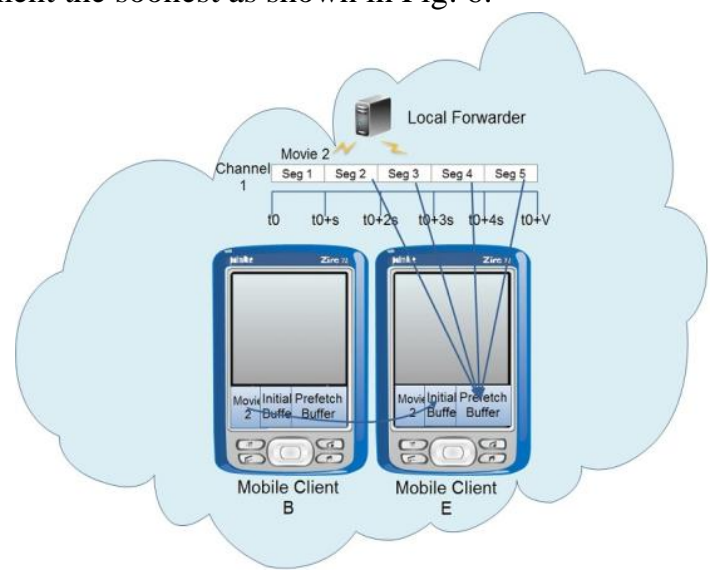

Fig. 6. Mobile client e requesting movie 2's $1^{\text {st }}$ segment from mobile client B.

d) Scenario of Playback procedure when the Clinet Request from neighbors:

1. U1 send request for S1 from neighbors who in the same area network of $\mathrm{U} 1$

2. U2 within the network area

3. U2 currently are not forwarding first segment to other mobile user

4. If such U2 exist

- Download S1 from U2 into U1's Initial_play buffer

- The U1 join the broadcasting channel and save the remaining segments of the video.

5. If download is complete

- U1 play data in its Initial_play buffer

- After play the S1, U1 switch to pre-fetched buffer to play remaining segments of the video.

6. Else If such U2 does not exist

7. Detect a local forwarder

8. Find the channel from local forwarder which is going to broadcast the $\mathrm{S} 1$ soonest

9. Wait and join that channel

10. Download the S1 in its Initial_play buffer and watch remaining segments from Pre-fetched buffer

11. Quit the channel.
The new client might not get the reply from all the neighbors so when the client get a reply from any of the neighbor, it will accept the 1 st segment from the neighbor that responded for the request without considering the distance of the neighbor. If the new client did not get any reply from its neighbors hence when the time reached until the time for the broadcasting protocol to broadcast the 1st segment again, the new client can immediately tune in to the channel that is going to broadcast the 1 st segment. If waiting time, twait = tdelay, tune in to the channel that is going to broadcast the 1st segment.

The new client can now start to watch the movie by playing the 1st segment from its initial buffer and can immediately switch to the pre-fetch buffer to continue watching the movie.

\section{ACKNOWLEDGMENT}

This project is part of a larger research project in the MCRG and this work was supported by Universiti RU grant (1001 / PKOMP/ 817066) from the Universiti Sains Malaysia.Last but not least, the authors would like to thank the School of Computer Science, Universiti Sains Malaysia (USM) for supporting this study.

\section{REFERENCES}

[1] D. A. Tran, M. Le, and K. A. Hua, "MobiVoD: A Video-on-Demand System Design for Mobile Ad hoc Networks," in Proceedings of the 2004 IEEE International Conference on Mobile Data Management (MDM'04), Berkeley, CA.

[2] A. A. Saleh and P. Sumari, "A Video on Demand System Architecture for Heterogeneous Mobile Ad Hoc Networks for Different Devices," 2010 2nd International Conference on Computer Engineering and Technology, vol. 7, 2012, pp. 700 - 707.

[3] K. Gorantala, "Routing Protocols in Mobile Ad-hoc Networks. Department of Computing Science," Sweden, Umea University.

[4] J. B. Kwon and H. Y. Heom, "Providing ver functionality in staggered video broadcasting," IEEE Transactions on Consumer Electronics, vol. 48, no. 1, pp. 41-48

[5] Y. Q. Gui, E. Jung, Y. Choi, and H. K. Choi, "An efficient periodic broadcast scheme for mobile video -on-demand system," in Proc. Of international Confernce on Information Technology (ITNG'07), pp. 888-889, 2007.

[6] M. Y. M. Chiu and K. H. A. Yeung, "Partial video sequence caching scheme for VOD systems with heterogeneous clients. Industrial Electronics," IEEE Transactions on, vol. 45, no.1, pp. 44-51, 1998.

[7] M. Dakshayini and T. Nair, "Client-to-Client Streaming Scheme for VOD Applications," The Internasional Journal of Multimedia and its Application (IJMA), vol. 2, no. 2, 2010.

[8] D. A. Tran, M. Le, and K. A. Hua, "MobiVoD: A Video-on-Demand System Design for Mobile Ad hoc Networks," in Proceedings of the 2004 IEEE International Conference on Mobile Data Management (MDM'04), Berkeley, CA.

[9] D. A. Tran, M. Le, and K. A. Hua, "MobiVoD: A Video-on-Demand System Design for Mobile Ad hoc Networks," in Proceedings of the 2004 IEEE International Conference on Mobile Data Management (MDM'04), Berkeley, CA.

[10] P. Branch, G. Egan, and B. Tonkin, "A client caching scheme for interactive video-on-demand. Networks," Proceedings. IEEE International Conference on. 1999. (ICON '99)

[11] P. Branch, G. Egan, and B. Tonkin, "A client caching scheme for interactive video-on-demand. Networks," Proceedings. IEEE International Conference on. 1999. (ICON '99)

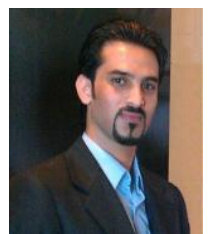

Saleh Ali Alomari obtained his Bachelor degree in Computer Science from Jerash University, Jordan in 2005 and Master degree in Computer Science from Universiti Sains Malaysia (USM), Pulau Penang, Malaysia in 2007. Currently, $\mathrm{He}$ is a $\mathrm{Ph}$. D. candidate at the School of Computer Science, Universiti Sains Malaysia. He is the candidate of the Multimedia 
Computing Research Group, School of Computer Science, USM. He is managing director of ICT Technology and Research and Development Division (RandD) in DandD Professional Consulting Company. He has published over 35 papers in international journals and refereed conferences at the same research area. He is a member and reviewer of several international journals and conferences (IEICE, ACM, KSII, JDCTA, IEEE, IACSIT, etc). His research interest are in area of Multimedia Networking, video communications system design, multimedia communication specifically on Video on Demand system, P2P Media Streaming, MANETs, caching techniques and for advanced mobile broadcasting networks as well.

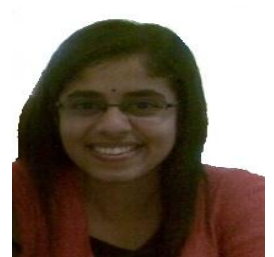

Vaithegy Doraisamy obtained her Bachelor degree in School of Computer Science from Universiti Teknologi Malaysia, Johor in 2008, and Master degree in Computer Science from Universiti Sains Malaysia (USM), Pulau Penang, Malaysia in 2010. Currently, she is a $\mathrm{PhD}$ candidate at the Multimedia Computing Research Group, School of Computer Science, Universiti
Sains Malaysia. Her research areas are in Video on Demand system, cloud computing and caching techniques.

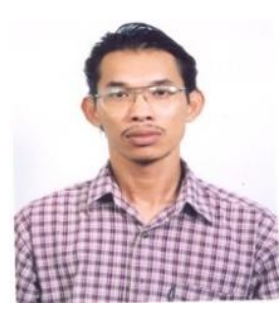

Putra Sumari obtained his $\mathrm{MSc}$ and $\mathrm{PhD}$ in 1997 and 2000 from Liverpool University, England. Currently, he is associate professor and lecturer at School of Computer Science, Universiti Sains Malaysia, Penang. Head of Multimedia Computing Research Group, School of Computer Science, Universiti Sains Malaysia, Pulau Penang. Member of ACM and IEEE, program committee and reviewer of several journals and International Conferences. He has published more than hundred papers including journal and conferences. His research areas are multimedia communication, specifically on video on demand system, content distributing network and image retrieval. 